\title{
Moyamoya disease associated with aortic coarctation
}

\author{
You-Min Zheng, Chun-Hong Xie, Fang-Qi Gong \\ Hangzhou, China
}

A 12-year-old girl was referred to our hospital due to fever, numbness on the left limb and movement disorder for three days. On admission, she had a body temperature of $37.5^{\circ} \mathrm{C}$, occasional cough, numbness on the left limb (severe in the left upper limb), motion limitation, difficulty to hold things with hands, drooping of the angle of the mouth, trembling of the mouth at times (persisting for seconds) and vomiting. No dizziness, blurred vision, diplopia, and tinnitus were found in the girl. Before admission, she was diagnosed with upper respiratory tract infection and gastritis and treated in a local hospital. She underwent an aortic angioplasty (resection of the narrow segment and end to end anastomosis) for correction of aortic coarctation at three years of age in our hospital; at 10 yReceived October 9, 2013 ears of age, percutaneous balloon aortic coarctation angioplasty was performed for re-coarctation (Fig. A \& B) of the aorta after surgery.

Physical examination showed a hypertension of $145 / 67 \mathrm{mmHg}$, rough breath sounds in the lungs, a $2 / 6$ systolic murmur, positive Babinski sign in the left limb, and grade $\mathrm{IV}^{+}$muscle force. Cranial MRI showed stenosis and occlusion of the anterior and middle cerebral arteries. Blood vessel flow-void-signal was found in the basal ganglia region (Fig. C). Magnetic resonance angiography revealed collateral circulation in network and long T1 and T2 signal intensity in the right temporal and parietal lobe. Moreover, cerebral infarction was found in the right temporal and parietal lobe (Fig. D). Dexamethasone was used to reduce the intracranial pressure, while nimoldipine was used to relieve vasospasm and oral aspirin to reduce

Author Affiliations: Department of Cardiology, Children's Hospital, Zhejiang University School of Medicine, Hangzhou 310003, China (Zheng YM, Xie CH, Gong FQ); Department of Pediatrics, Taizhou Hospital, Wenzhou Medical College, Linhai 317000, China (Zheng YM)

Corresponding Author: Chun-Hong Xie, 57 Zhuganxiang, Hangzhou 310003, China (Tel: 86-571-88873691; Fax: 86-571-87033296; Email: xch_hz@hotmail.com)

doi: 10.1007/s12519-014-0516-7

(C)Children's Hospital, Zhejiang University School of Medicine, China and Springer-Verlag Berlin Heidelberg 2014. All rights reserved.

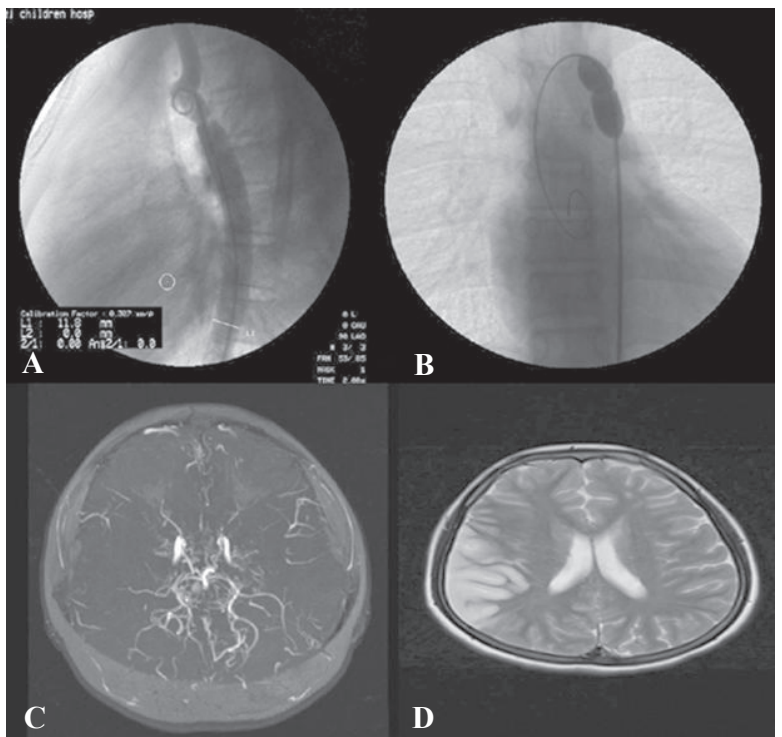

Fig. A: Descending aorta angiography showing aortic coarctation in the patient; B: Percutaneous balloon aortic coarctation angioplasty showing "waist" of the balloon; C: Magnetic resonance angiography showing occlusion of both sides of the middle cerebral artery, of which the distal branches did not show. Both sides of the posterior cerebral artery show compensatory thickening, expanding, and multiple fine reticular vascular tortuosity in the basis cranii and basal ganglia; D: Long T1 and T2 signal intensity in the temporal and parietal lobe.

anticoagulation. Symptoms were improved after three days of treatment and the patient was discharged from the hospital after two weeks. She had no numbness of the limbs with grade $\mathrm{V}$ muscle force in the left limbs on discharge.

\section{Funding: None.}

Ethical approval: The consent to report this case has been obtained from the parents of the patient.

Competing interest: The authors declare that they have no competing interests.

Contributors: ZYM participated in data collection and drafted the manuscript. XCH and GFQ participated in study design and drafting of the manuscript. All authors read and approved the final manuscript.

Received August 26, 2014 Accept after revision September 4, 2014 